Rapports médicaux uniformisés pour l'assurance accidents, l'assurance maladie / indemnités journalières et l'assurance responsabilité civile

\title{
Simplifier les travaux administratifs
}

\section{Bruno Soltermann}

Médecin chef de l'Association Suisse d'Assurances ASA
A des fins d'uniformisation, l'Association Suisse d'Assurances a élaboré ou actualisé les rapports médicaux pour l'assurance-accidents, l'assurance-maladie/indemnités journalières et l'assurance responsabilité civile. Tous les assureurs privés suisses utilisent dès maintenant ces nouveaux rapports et souhaitent ainsi répondre à la demande du corps médical de simplifier les travaux administratifs.

On distingue deux groupes de formulaires: le «Rapport médical initial» et le «Rapport médical intermédiaire». Dans le cadre de l'assurance-accidents, les deux rapports médicaux sont utilisables autant dans le domaine de l'assurance obligatoire selon la LAA que dans le domaine de l'assurance complémentaire facultative selon la loi sur le contrat d'assurance (LCA). C’est le cas également pour l'assurance-maladie obliga- vail conformément aux certificats différenciés d'incapacité de travail de la Swiss Insurance Medicine ou des diverses sociétés médicales cantonales.

Tous les formulaires sont rédigés en français, en allemand et en italien, et disponibles sous forme électronique à remplir directement (champs variant selon les besoins) ou à imprimer et remplir (champs déterminés).

Les rapports médicaux peuvent être consultés et également téléchargés sous www.svv.ch/fr/medecine/ formulaires sur le site Internet de l'ASA, mais en règle générale l'assureur envoie le formulaire à remplir au médecin.

En ce qui concerne l'utilisation des rapports médicaux, nous prions tous les médecins de prêter attention aux trois dispositions suivantes:

\section{«ll ne devrait avoir plus aucun problème de protection des données lié au transfert des données entre le médecin et les assureurs privés.»}

toire et indemnités journalières selon la LAMal et aussi selon la LCA (majorité des assurances indemnités journalières et assurance-maladie complémentaire). Pour la responsabilité civile dont la couverture n'est pas subdivisée, le rapport médical est analogue à celui de l'assurance-accidents.

Il est possible de déclarer de manière différenciée dans tous les rapports médicaux les incapacités de tra-
Correspondance:

Dr Bruno Soltermann

Association Suisse d'Assurances

Conrad-Ferdinand-Meyer-Strasse 14

Case postale

CH-8022 Zurich

bruno.soltermann[at]svv.ch
- Une procuration n'est pas nécessaire dans le cadre de la loi sur l'assurance-accidents (LAA): le rapport médical est envoyé directement à l'assureur accidents, en général à la personne responsable du cas (LAA, art. 54a).

- Une procuration n'est également pas nécessaire dans le cadre de l'assurance-maladie (LAMal) qui concerne surtout les prestations de soins et en marge les indemnités journalières selon la LAMal. Le rapport médical est envoyé au médecin-conseil de l'assureur-maladie (LAMal, art. 57).

- Dans tous les autres cas (assurance complémentaire selon la LCA pour la LAA ou pour la LAMal, indemnités journalières selon la LCA, responsabilité civile), une procuration est nécessaire et le rapport médical est envoyé directement à l'assureur, en général à la personne responsable du cas.

Maintenant que le titre des rapports médicaux indique clairement le domaine d'assurance pour lequel l'assureur demande un rapport médical, il ne devrait avoir plus aucun problème de protection des données lié au transfert des données entre le médecin et les assureurs privés.

Dans le cadre de la LAA et de la LAMal, les honoraires pour l'établissement de rapports médicaux sont déterminés par TARMED, dans tous les autres cas ils sont convenus directement avec l'assureur.

Les rapports médicaux peuvent être téléchargés sur le site Internet de I'ASA, mais en règle générale l'assureur envoie le formulaire à remplir au médecin. 